\title{
ActiveBelt: Belt-type Wearable Tactile Display for Directional Navigation
}

\author{
Koji Tsukada ${ }^{1}$ and Michiaki Yasumura ${ }^{2}$ \\ 1 Graduate School of Media and Governance, Keio University, 5322 Endo Fujisawa, \\ Kanagawa 252-8520, Japan, \\ tsuka@sfc.keio.ac.jp, \\ http://mobiquitous.com/index-e.html \\ 2 Faculty of Environmental Information, Keio University, \\ yasumura@sfc.keio.ac.jp.
}

\begin{abstract}
In this paper we propose a novel wearable interface called "ActiveBelt" that enables users to obtain multiple directional information with the tactile sense. Since the information provided by the tactile sense is relatively unobtrusive, it is suited for daily use in mobile environments. However, many existing systems don't transmit complex information via the tactile sense. Most of them send only simple signals, such as vibration in cellular phones. ActiveBelt is a novel belt-type wearable tactile display that can transmit directional information. We have developed prototype systems and applications, evaluated system performance and usability, and demonstrated the possibility of practical use.
\end{abstract}

\section{Introduction}

As positioning technologies such as GPS (Global Positioning System) have become popular and widely used, many researchers have developed location-aware information systems in mobile environments using positioning technologies[3] [14][24]. Moreover, in Japan, some cell phone companies have provided locationaware information services, such as "eznavigation" [7] by KDDI.

While there are many applications for these location-aware information services, one of the most popular applications is navigation[11]. For example, "eznavigation" provides an application called "GPS Map" that can display a fairly accurate map of the current location in a few seconds using GPS and information of base stations. These navigation systems look useful at first glance. However, users often have difficulty matching geographical information on maps to the real world. For example, people often get lost in a large exhibition even if they have a map of it. Moreover, some people are not good at reading maps.

Since these problems result from a difficulty to match geographical information on 2D maps to the real world, we can address the problems by providing suitable directional information to a destination. For example, when a person gets lost in a large exhibition, it's very helpful for someone to tell her/him what the correct direction to her/his destination is. 
In this paper we propose a novel belt-type wearable interface called "ActiveBelt" that enables users to obtain directional information via the tactile sense. First, we describe properties of tactile displays and belt-type devices.

\subsection{Types of Display in Mobile Environments}

Three types of display used in mobile and wearable computing are: visual displays, auditory displays, and tactile displays. Each of these types of display has advantages and disadvantages. The visual display can transmit vast quantities of information quickly, but it prevents visual attention being directed to other tasks. For example, a user often has difficulty looking at a display of a cell phone while walking. The Auditory display is more easily used with other tasks. However, the auditory display can conflict with other sounds in the surrounding environment. For example, it is difficult for users to use the auditory display in very noisy or silent environments. The tactile display is limited in the amount of information it can transmit. However, it's easiest for users to use the tactile display with other tasks, and so it is suited for daily use in mobile environment.

In this paper we focus on the tactile display as a display type that is well suited to mobile use.

\subsection{Types of Forms of Wearable Interfaces}

As shown in Table 1, many researchers proposed various forms of wearable interfaces such as glasses, gloves, watches, rings, shoes, vests, pendants, caps, earrings, and so on. We have focused on a belt-type device since the shape of a belt seems suited to transmitting directional information via the tactile sense. Since a belt is worn around the user's torso, it can provide directional information only by activating vibrators. Moreover, since many people usually wear belts, they don't need to wear additional devices. In addition, although many researchers in wearable computing used belts for attaching computers and batteries, the possibility of belts for input/output interfaces has not received much attention. Because of these considerations, we chose a belt-type device.

\section{ActiveBelt}

\subsection{Concepts}

In this section we describe the concepts of the ActiveBelt. The main concepts of the ActiveBelt are as follows:

1. Tactile display that enables users to intuitively obtain directional information

2. Belt-type wearable device optimized for mobile use

3. Suited for various applications, especially for location-aware information services. 
Table 1. Typical products and research projects of wearable interfaces classified by their forms

\begin{tabular}{c||l}
\hline Forms & Typical products and research projects \\
\hline \hline glasses & MicroOptical[13], Poma[17] \\
\hline gloves & 5DTDataGlove[1], CyberGlove[6], Ubi-Finger[25] \\
\hline watches & Whisper[10] \\
\hline rings & FingeRing[9], GSR rings[16] \\
\hline shoes & Expressive Footwear[15], GSR Shoes[16] \\
\hline vests & Tactual Wearable Display[23] \\
\hline pendants & GesturePendant[21] \\
\hline caps & Wearable American Sign Language[22] \\
\hline earrings & The Blood Volume Pressure earring[16] \\
\hline
\end{tabular}

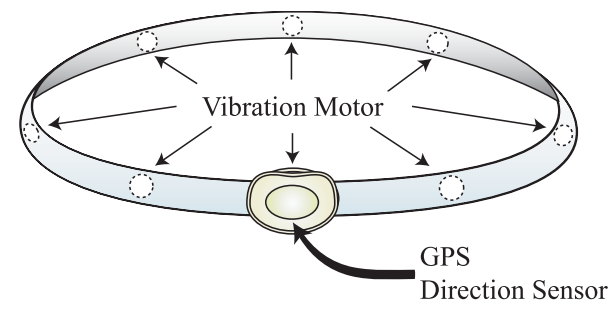

Fig. 1. Basic Concept of ActiveBelt

First, the ActiveBelt can enable users to intuitively obtain directional information in the real world simply by activating vibrators. Since a user can easily match this tactile information to directions in the real world, the ActiveBelt can transmit effective information via the tactile sense.

Second, the ActiveBelt doesn't require the user to wear or carry yet another device, since it attaches vibrators in a typical belt worn by many people in daily life. In addition, since people usually wear belts outside, ActiveBelt is well suited to use in mobile environments.

Third, the ActiveBelt can be used for a variety of applications and especially for location-aware information services like navigation systems.

\subsection{Device Architecture}

In this section, we describe the device architecture of the ActiveBelt. The ActiveBelt consists of two sensors (a direction sensor and a GPS) to detect a user's location and orientation, multiple vibrators to transmit tactile information, and a microcomputer to control these devices (Fig. 1). The vibrators are attached throughout a belt at regular intervals. 


\subsection{Sensitivity of the Torso to Vibration}

In this section, we discuss the sensitivity of the torso to the vibration. According to van Erp[26], the resolutions of the torso are as follows: In the abdominal area, the resolution has a roughly $0.4 \mathrm{~cm}$ jnd(just noticeable difference) interval at the center, and a $1.2 \mathrm{~cm}$ interval at the sides. In the back, the resolution is less sensitive about a $1.8 \mathrm{~cm}$ interval at the center, and about a $3.0 \mathrm{~cm}$ interval at the sides. These results show that the resolutions of a torso are fairly good. Moreover, Yamamoto[30] reported that people can distinguish horizontal direction fairly accurately based on vibration to their torsos.

Thus, the resolutions of a torso are fairly good, and people can tell horizontal direction with vibration. This suggests that the ActiveBelt may help navigation using vibration to indicate the direction to take at each point in time.

\subsection{Numbers of Vibrators to be Used}

Human sensitivity allows for the use of a relatively large number of vibrators around the torso. However, too many vibrators may generate problems, such as increases in power consumption, complications of cables, needs for more I/O of microcomputers, and so on.

To avoid these problems, we used eight vibrators. These vibrators and cables can be easily attached inside a belt, and controlled using a microcomputer.

\section{Development}

\subsection{Prototype}

We developed a prototype ActiveBelt system based on the above concepts. The prototype system consists of four components: (1)ActiveBelt hardware, (2)a GPS, (3)a directional sensor, and (4) a microcomputer (Fig. 2). Fig. 3 shows the system architecture of the ActiveBelt. Next, we explain each component of the prototype system.

Belt-type hardware We developed the first prototype of the ActiveBelt in the form of a typical belt adjusted using a buckle (Fig. 4). We attached eight vibrators (FM23A by TPC) and LEDs ${ }^{3}$ in the belt. The diameter of the FM23A is about $18 \mathrm{~mm}$, and the thickness is about $3 \mathrm{~mm}$. We attached four vibrators in the center of the abdomen, in the center of the back, and the left and right sides close to the hips. We also attached four other vibrators in each midway between two of the first four vibrators.

The frequencies of each vibrator change with voltages, such as $33 \mathrm{~Hz}$ at 0.3 $\mathrm{V}, 40 \mathrm{~Hz}$ at $0.6 \mathrm{~V}, 55 \mathrm{~Hz}$ at $0.8 \mathrm{~V}$, and $77 \mathrm{~Hz}$ at $1.2 \mathrm{~V}$. Thus, we can control the

\footnotetext{
${ }^{3}$ LEDs are turned on and off in sync with vibrators. We used LEDs to check the states of the ActiveBelt.
} 


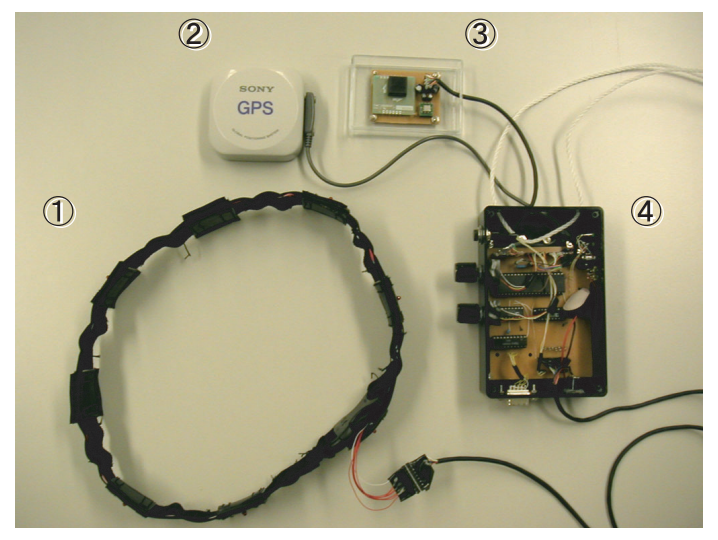

Fig. 2. Prototype system of ActiveBelt (1. ActiveBelt hardware, 2. GPS, 3. directional sensor, 4. microcomputer)

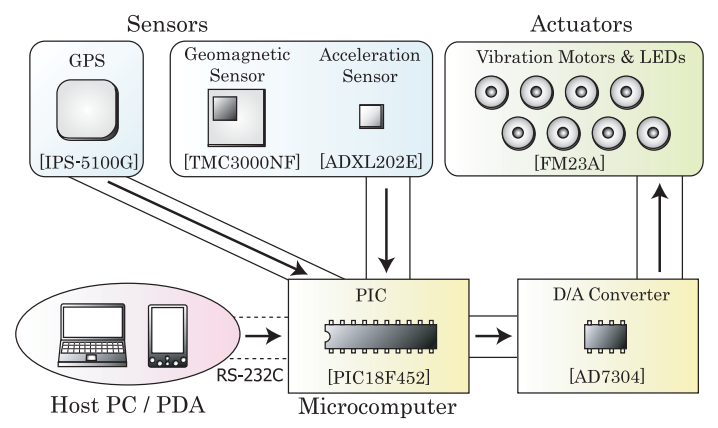

Fig. 3. System architecture of ActiveBelt

strength of vibrators by changing the voltages ${ }^{4}$. For human skin, the most sensitive frequency band is between $200 \mathrm{~Hz}$ and $250 \mathrm{~Hz}$. We used a less sensitive frequency band between $33 \mathrm{~Hz}$ and $77 \mathrm{~Hz}$ in the prototype system. We focused on using cheap and easily obtainable vibrators this time. Other vibrators may be used in later research.

We adjusted the length of the belt to the waist size of one of the authors. The distance between the vibrators was $9.75 \mathrm{~cm}$ on average $( \pm 0.25 \mathrm{~cm})$, and the whole length of the belt was about $78.0 \mathrm{~cm}$. It was difficult for many users to wear this prototype, since the mapping between vibrators and directions was modified when a user adjusted the length of the belt to her/his waist.

To solve this problem, we developed a universal-size ActiveBelt ver.2 that consists of leather parts in which vibrators are attached and elastic rubber parts

\footnotetext{
${ }^{4}$ Since energies change with frequencies in general, people feel stronger vibration in a high frequency.
} 


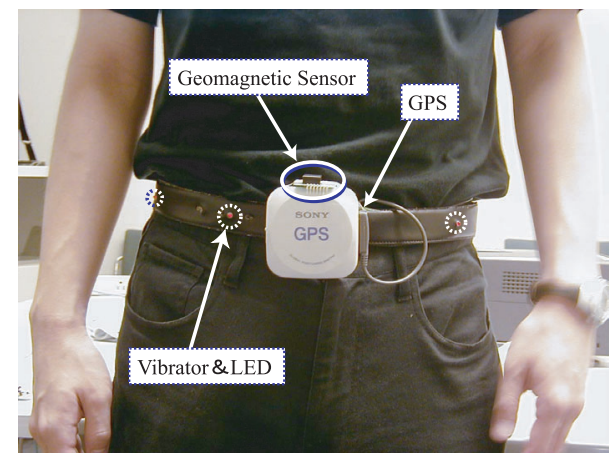

Fig. 4. Hardware of ActiveBelt ver.1

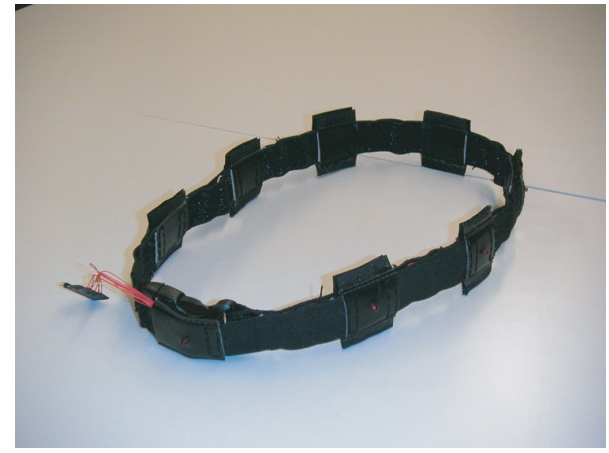

Fig. 5. Hardware of ActiveBelt ver.2 (universal-size)

fit to the size of the user's waist (Fig. 5). While the whole length of the belt is about $75.0 \mathrm{~cm}$, a wide range of users with waist circumference less than 98.0 $\mathrm{cm}$ can wear the belt without breaking the correspondences between vibrators and directions (Fig. 7) . We have wired each vibrators loosely in consideration of elasticity of rubber parts (Fig. 6).

Directional Sensor and GPS The directional sensor consists of a geomagnetic sensor (TMC3000NF by NEC Tokin) and an acceleration sensor (ADXL202E by Analog Devices)(Fig. 8). We used the geomagnetic sensor to detect the orientation of a user. The output voltages of the geomagnetic sensor are converted to digits with 10 bit $\mathrm{A} / \mathrm{D}$ converters in a microcomputer. The accuracy of the geomagnetic sensor for detecting directions is about \pm 1.5 degree. We used the acceleration sensor to detect horizontal angles. We considered using the acceleration sensor for calibrating the horizontal angles of the geomagnetic sensor, since the geomagnetic sensor must keep its position horizontal to detect precise directions. However, through simple experiment, we found that humans can eas- 


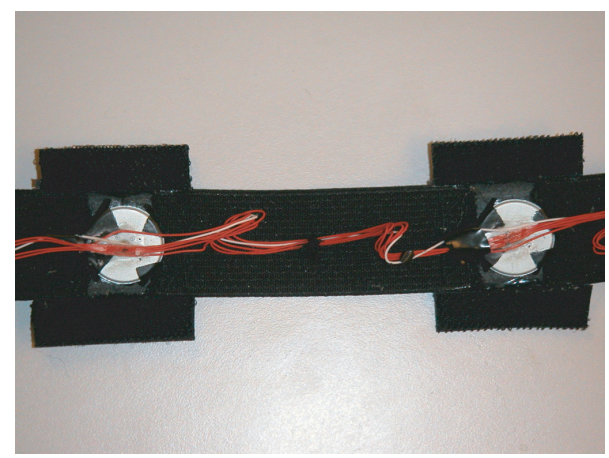

Fig. 6. Wiring inside of universal-size ActiveBelt

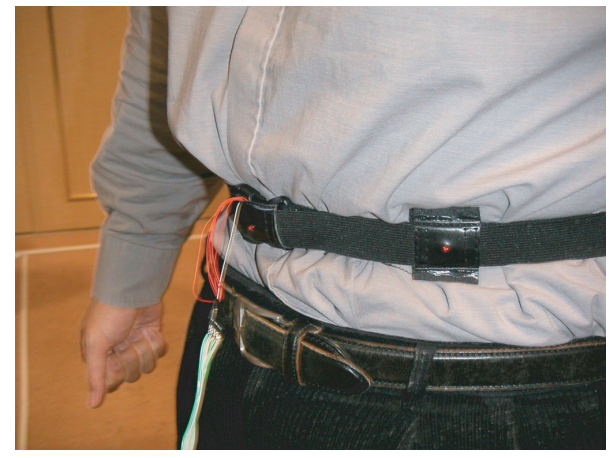

Fig. 7. Example of wearing universal-size ActiveBelt

ily keep the position of their waist horizontal even when they walk on ascents of about 30 degrees. In addition, perfect precision is not needed. Consequently, we don't modify the outputs of the geomagnetic sensor in the current prototype system.

We used the IPS-5100G (by Sony) as a GPS. The IPS-5100G transmits output signals to the microcomputer as serial data, and the microcomputer extracts latitudes and longitudes from the data. We used spherical trigonometry to calculate a relative direction and distance to a destination.

Microcomputer and Surrounding Circuit We used a microcomputer (PIC $18 \mathrm{~F} 452$ by MicroChip) to control the sensors and vibrators. The functions of the microcomputer are as follows: (1) controlling vibrators and LEDs based on input data from sensors, and (2) communicating with host PC/PDA. For the first function, the microcomputer controls the vibrators and LEDs with a D/A converter (AD7304 by Analog Devices). Since we can easily control output voltages using the D/A converter, we can control the frequencies of the vibrators 


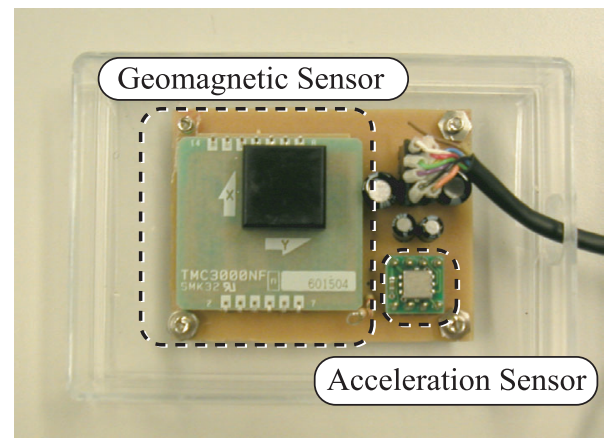

Fig. 8. Circuit board of direction sensor

and the brightness of the LEDs smoothly ${ }^{5}$. For the second function, the microcomputer communicates with the host PC/PDA via RS-232C. Users can register the latitude and longitude of a destination with the host PC/PDA.

\subsection{Applications}

In this section, we discuss applications of the ActiveBelt. We propose four applications: (1) FeelNavi for human navigation systems, (2) FeelSense for locationaware information services, (3) FeelSeek for search of lost properties, (4)FeelWave for entertainment.

FeelNavi FeelNavi is an application of navigation using tactile information. Users can intuitively reach destinations by walking toward the direction of vibration. In the prototype system, a user registers the latitude and longitude of her/his destination with a host PC/PDA. The ActiveBelt then activates the specified vibrator, based on the current position and orientation of the user (Fig. 9). We express a distance to a destination using pulse intervals of vibration. When a user comes nearer to the destination, the pulse intervals of vibration become shorter.

FeelSense As mentioned before, many researchers and companies have developed location-aware information services. Most of these existing systems used visual displays. Although the visual display has an advantage that it can transmit vast quantities of information quickly, this approach is sometimes unsatisfactory for users. Moreover, location-aware information services are expected to extend rapidly in the near future. For these reasons, we should consider new approaches that are more convenient for users.

${ }^{5}$ The voltage range we used was between $0 \mathrm{~V}$ and $1.2 \mathrm{~V}$. We could activate vibrators by raising the voltage above $0.3 \mathrm{~V}$. 


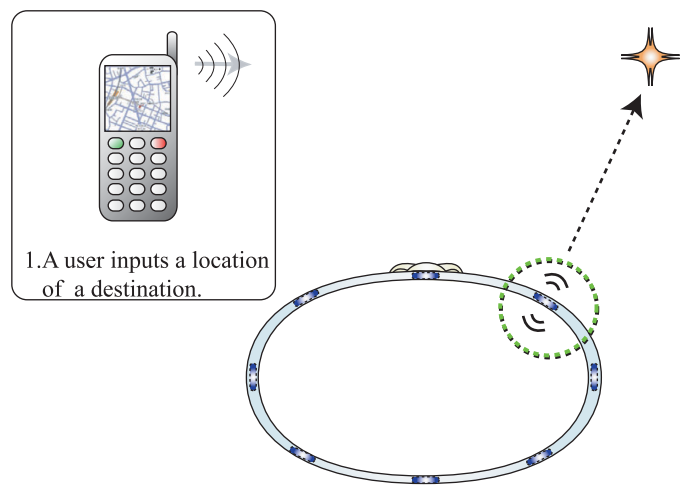

2.ActiveBelt Transmits direction and distance to the destination with tactile sense.

Fig. 9. Basic concept of FeelNavi

FeelSense is an always-active tactile display with which users can detect location-aware information while walking around a town. As shown in Fig. 10, users register interesting categories in advance. When they come near to relevant location-aware information, the ActiveBelt activates specified vibrators based on a user's position and orientation, and the location of the target information. For example, they can easily find shops of interest such as boutiques or restaurants.

FeelSeek Recently, RFID (Radio Frequency Identification) tags have become smaller and cheaper, and they are expected to be attached in various commodities[29]. Moreover, a real-world reminder system using proximity information is a useful application in ubiquitous environments[12]. In such a situation, it is useful to attach RFID tags to valuables such as wallets and cell phones, and to tell users immediately if they leave their valuables behind.

FeelSeek is an application to remind users of valuables left behind, and to navigate users toward those valuables. As shown in Fig. 11, when the RFID tagged object moves away from the RFID reader attached on the belt, the ActiveBelt activates all vibrators to remind a user of it. Then, she/he inputs a trigger information if she/he want to search it. Finally, the ActiveBelt calculates a brief position of the item using records of GPS, and navigates the user to it.

FeelWave FeelWave is an application to amuse users with rhythmic vibration in sync with music (Fig. 12). For example, since ActiveBelt can transmit tactile information in all direction, it can be used as a stereophonic tactile display with stereophonic music. In addition, the ActiveBelt may be used as new expressive medium such that it transmits rhythmic vibrations to members of an audience in sync with a turntable played by a DJ. 


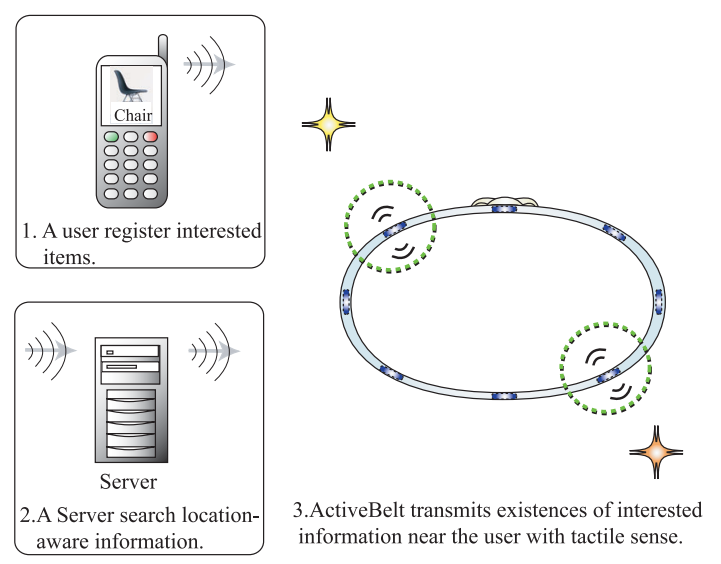

Fig. 10. Basic concept of FeelSense

\section{Evaluation}

We have evaluated the effectiveness of the ActiveBelt using the universal-size prototype system. As mentioned above, in the application of a human navigation system called FeelNavi, we express the direction to a destination by activating a specific vibrator and express the distance by controlling pulse intervals of vibration. In this evaluation, we examined whether subjects can recognize changes of direction, and pulse intervals of vibration, both in a standing state and in a walking state. We selected six test subjects who had never used the ActiveBelt before. Their ages ranged between 21 and 30 .

\subsection{Methods}

The subjects wore the universal-size ActiveBelt and a wireless RS-232C transceiver. The experimenter controlled the direction and interval of vibration with a PDA and a wireless RS-232C transceiver(Fig. 13). The experimenter activated only one vibrator at a time, and kept the strength of vibration at about $77 \mathrm{~Hz}$. The period of each evaluation was about 15 minutes.

There were two postural conditions in the study: standing, and walking. In the standing condition, the experimenter controlled the direction of vibration and asked the subjects to indicate the direction orally or with gestures. We used four pulse intervals of vibration, that is, $250 \mathrm{msec}$ (125msec On, $125 \mathrm{msec}$ Off), $500 \mathrm{msec}(250 \mathrm{msec}$ On, $250 \mathrm{msec}$ Off), $1000 \mathrm{msec}(500 \mathrm{msec}$ On, $500 \mathrm{msec}$ Off), and $1680 \mathrm{msec}(840 \mathrm{msec}$ On, $840 \mathrm{msec}$ Off). In the walking condition, the experimenter controlled the direction of vibration and asked the subjects to turn to the direction of vibration. We used the same four pulse intervals of vibration as described above. 


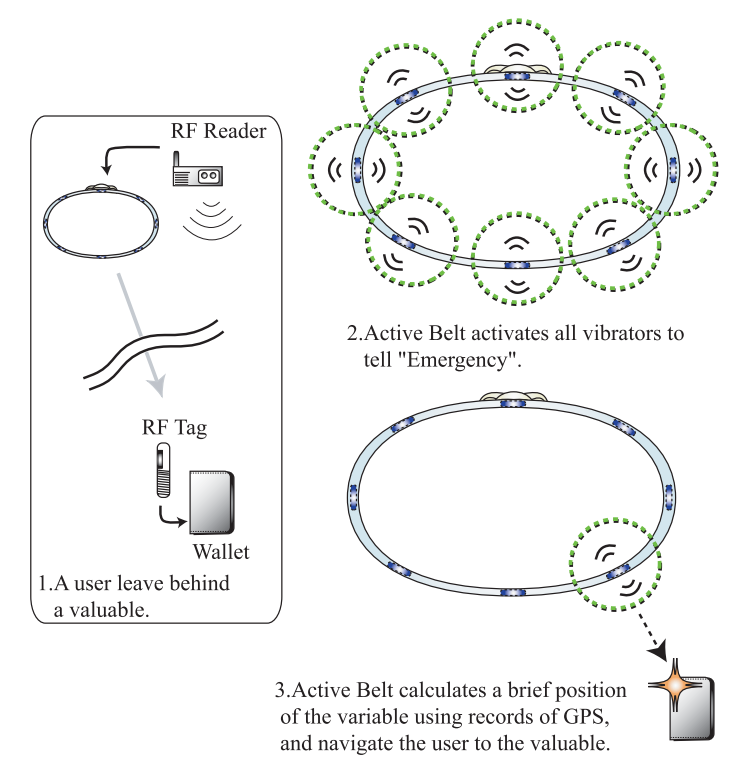

Fig. 11. Basic concept of FeelSeek

The experimenter observed the behaviors of subjects during each condition. After each condition, the experimenter asked them questions to obtain their subjective feedbacks about changes in direction and pulse intervals signalled by the vibration. At the end of the evaluation, the experimenter asked each of them about their impressions of the prototype system including what problems they experienced.

\section{$4.2 \quad$ Results}

In the standing condition, all subjects were able to recognize all changes in the four pulse intervals. For changes in direction, five subjects were able to tell direction to a fair degree of accuracy within about one second. We asked the subjects "Can you differentiate the eight directions in each pulse interval?". Five subjects said they could easily tell the difference amongst all directions for all intervals. On the other hand, one subject said that he could easily recognize four directions (front, back, left, and right) in all intervals, but could not easily recognize the other four directions. This difficulty may have resulted from his waist being very thin (about $67 \mathrm{~cm}$ ). Since the belt did not fit with his waist, the ActiveBelt was not able to transmit enough tactile information.

In the walking condition, the behaviors of the subjects were quite different for each pulse interval. In the case when the pulse intervals were $1680 \mathrm{msec}$ and $1000 \mathrm{msec}$, when the experimenter changed a direction of vibration, all subjects walked for one or two more steps, stopped for a moment, and then turned to 


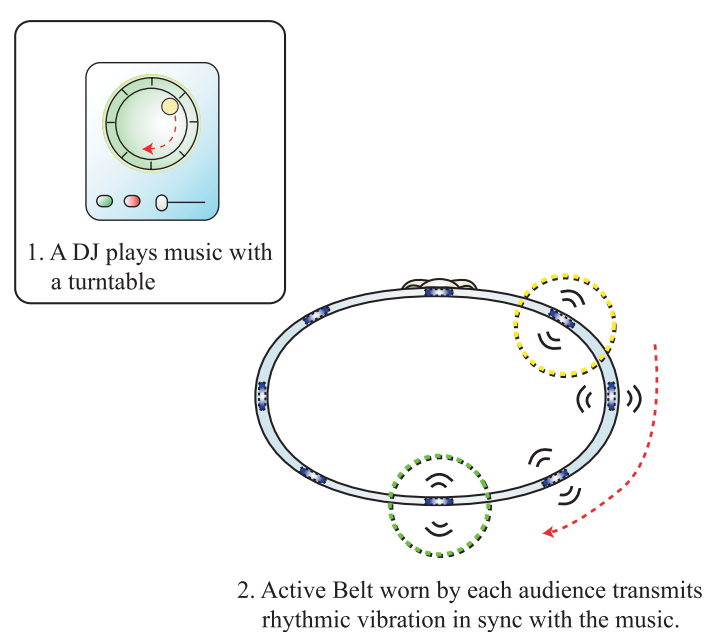

Fig. 12. Basic concept of FeelWave

the direction of vibration. When questioned, they said that they were able to recognize the changes in vibration while walking, and that they were able to recognize the direction if they stopped for a moment. For the pulse intervals of $500 \mathrm{msec}$, all subjects sometimes failed to recognize the change of vibration. Two subjects said that they were not able to feel vibration in this range. When the pulse intervals were $250 \mathrm{msec}$, all subjects said that they were not able to feel vibration.

Finally, we summarize the impressions that subjects reported about the prototype system.

1. Vibration on the back is perceived to be weaker than vibrations on the abdomen. $(5 / 6)$

2. For practical use, it is more desirable that the vibrators are activated only when users get lost than that they are always activated. (2/6)

3. For practical use, eight vibrators are not necessarily needed, and four vibrators are good enough. $(3 / 6)$

\subsection{Consideration}

Based on the above results, we discuss current problems and propose improvements needed for practical use of the ActiveBelt.

Providing Direction When standing, almost all subjects were able to recognize directions within about one second. When walking, and for the pulse intervals of more than $1000 \mathrm{msec}$, all subjects recognized changes of vibration within two steps, stopped only for a moment, and then turned to the direction 

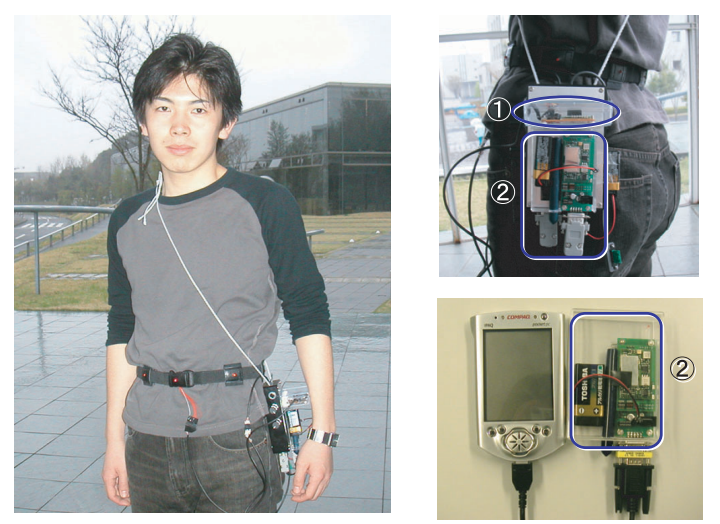

Fig. 13. Prototype system used in the evaluation. left: a subject, upper right: a microcomputer and surrounding circuit, lower right: a controller for the experimenter (1. a directional sensor, 2.a wireless RS-232C transceiver)

of vibration. Thus, we confirmed that the ActiveBelt can navigate users to the target direction using vibration both when standing and when walking.

Indicating Distance In FeelNavi, we indicated distance to a destination using pulse intervals of vibration. When standing, all subjects were able to recognize changes in the four pulse intervals. However, when walking, they often failed to recognize vibration with a pulse less than $500 \mathrm{msec}$. We will examine other expressions of distance, along with the pulse intervals.

Navigating In oral interviews, we received interesting feedbacks about navigation. First, some subjects said that it is more desirable that the vibrators are activated only when users get lost than that they are always activated. This approach looks similar to behaviors that people pick up and look at maps only when they get lost.

Some subjects said that they thought four vibrators were good enough for navigation. However, we think it is useful to attach eight or more vibrators, since higher resolutions are useful for versatile applications and for error correction.

\section{Discussion}

Most existing human navigation systems in mobile environments apply visual displays, that is, they plot users' positions and orientations on 2D displays of PDAs or cell phones. We discuss here the differences between visual navigation systems and the belt-type tactile navigation system described in this paper.

The merits of visual navigation systems are as follows:(1) visual navigation systems can easily transmit complex information such as the layout of buildings 
near the destination and a detailed route to it, and (2)they usually provide input interfaces, so users can easily use them interactively.

The merits of the belt-type tactile navigation system are as follows: (1)the belt-type tactile navigation system is convenient to use when walking since it doesn't occupy users' visual or auditory senses, (2)users can intuitively recognize directions to their destinations even when the they are not good at reading maps, and (3)it can navigate users to their destinations even in bad conditions such as in heavy fog or deep night, or with no landmarks near locations of users, since it doesn't depend on visual landmarks in the surrounding environment.

Each system, visual and tactile, has its own merits. We will improve our system by combining the merits of visual and tactile navigation systems.

\section{Related Work}

Other research on human navigation systems and location-aware information services includes Walk Navi[14], Augument-able Reality[19], Touring Machine[8], Cyberguide[3], and Space Tag[24]. Various applications with location-aware information have been proposed, and most of the associated systems used visual displays.

A cell phone equipped with a GPS and a directional sensor[2] can display a map of a user's position, and rotate the map in sync with the orientation of the cell phone. This approach is interesting in helping users to match 2D maps and real world using directional information.

Research has been carried out on wearable tactile interfaces such as Cyber Touch, Tactual Wearable Display and GentleGuide. Cyber Touch[6] can generate tactile feedbacks of objects in virtual world, using six vibrators attached on gloves. Tactual Wearable Display[23] attached a matrix of vibrators to the back of a vest, and tried to transmit directions and other information. It also proposed an interesting tactile system using cognitive characteristics of the tactile sense such as apparent movement ${ }^{6}$. GentleGuide[4] proposed a tactile navigation system via two bracelets. It outputs three commands, left, right, and stop(activate both bracelets). In the meanwhile, our approach used a belt-type tactile display, and can enable users to obtain directional information more intuitively with the tactile sense.

There is research on tactile displays in aircrafts cockpits[18][20][27]. These research projects used vest-type tactile displays to provide pilots with navigation information. Brewster proposed a sophisticated conceptual framework for tactile displays and information presentation[5]. This approach is interesting in discussing many parameters used for designing tactile displays.

Waist Measure Belt[28] is a unique belt-type wearable device. A user can easily measure her/his waist-size only by wearing it which contains a digital

\footnotetext{
${ }^{6}$ When a user is stimulated on two point of her/his skin alternatively in a specified interval, she/he feels continuous stimulations as if a small animal is hopping between the two points.
} 
measuring scale. It is interesting in proposing an application of health monitoring using a belt-type device.

\section{Conclusion}

We have proposed a novel belt-type wearable interface called "ActiveBelt" that enables users to obtain multiple directional information with the tactile sense. We developed prototype systems, and evaluated the possibilities for practical use. When we solve the problems of the current prototype systems, we believe that ActiveBelt will become a useful and intuitive navigation system. We are also examining other applications for entertainment and location-aware information services.

\section{References}

1. 5DT Data Gloves: http://www.5dt.com/hardware.html\#glove.

2. A cell phone equipped with a GPS and a directional sensor: http://www.kyocera.co.jp/prdct/telecom/consumer/a5502k/ez_navi.html (in Japanese).

3. Abowd, G. D., Atkeson, C. G., Hong, J., Long, S., Kooper, R. and Pinkerton, M.: Cyberguide: a mobile context-aware tour guide, Wireless Networks, Vol. 3, No. 5, pp. 421-433 (1997).

4. Bossman, S., Groenendaal, B., Findlater, J. W., Visser, T., de Graaf, M. and Markopoulos, P.: Gentleguide: An exploration of haptic output for pedestrian guidance, Proceedings of the Mobile HCI, 8-10-2003, Springer Publications, pp. 358-362 (2003).

5. Brewster, S. and Brown, L.: Tactons: Structured Tactile Messages for Non-Visual Information Display, Proceedings of Australasian User Interface Conference 2004, pp. 15-23 (2004).

6. CyberGlove and Cyber Touch: http://www.immersion.com/products/3d/.

7. Eznavigation: http://www.keitai1bankan.com/phone/ez_navi.html (in Japanese).

8. Feiner, S., MacIntyre, B., Hollerer, T. and Webster, A.: A Touring Machine: Prototyping 3D Mobile Augmented Reality Systems for Exploring the Urban Environment, Proceedings of First International Symposium on Wearable Computers(ISWC'97), pp. 74-81 (1997).

9. Fukumoto, M. and Tonomura, Y.: Body coupled FingeRing: Wireless wearable keyboard, Proceedings of the ACM Conference on Human Factors in Computing Systems(CHI'97), Addison-Wesley, pp. 147-154 (1997).

10. Fukumoto, M. and Tonomura, Y.: Whisper: a wristwatch style wearable handset, Proceedings of the ACM Conference on Human Factors in Computing Systems (CHI'99), pp. 112-119 (1999).

11. Holland, S., Morse, D. R. and Gedenryd, H.: AudioGPS: Spatial Audio Navigation with a Minimal Attention Interface, Personal and Ubiquitous Computing, Vol. 6, No. 4, pp. 253-259 (2002).

12. Lamming, M. and Bohm, D.: SPECs: Another Approach to Human Context and Activity Sensing Research, Using Tiny Peer-to-Peer Wireless Computers, Ubicomp 2003, Springer Publications, pp. 192-199 (2003). 
13. MicroOptical: http://www.microopticalcorp.com/.

14. Nagao, K. and Rekimoto, J.: Agent Augmented Reality: A Software Agent Meets the Real World, Proceedings of Second International Conference on Multi-Agent Systems(ICMAS'96), pp. 228-235 (1996).

15. Paradiso, J. and Hu, E.: Expressive Footwear for Computer-Augmented Dance Performance, Proceedings of First International Symposium on Wearable Computers(ISWC'97), pp. 165-166 (1997).

16. Picard, R. and Healey, J.: Affective Wearables, Personal Technologies, Vol. 1, No. 4, pp. 231-240 (1997).

17. Poma: http://www.xybernaut.com/Solutions/product/poma_product.htm.

18. Raj, A., Kass, S. and Perry, J.: Vibrotactile displays for improving spatial awareness, Proceedings of the Human Factors and Ergonomics Society Annual Meeting, Springer Publications, pp. 181-184 (2000).

19. Rekimoto, J., Ayatsuka, Y. and Hayashi, K.: Augment-able Reality: Situated Communication through Physical and Digital Spaces, Proceedings of Second International Symposium on Wearable Computers (ISWC'98), pp. 68-75 (1998).

20. Rupert, A.: Tactile situation awareness system: proprioceptive prostheses for sensory deficiencies, Space and Environmental Medicine 71, pp. 92-99 (2000).

21. Starner, T., Auxier, J., Ashbrook, D. and Gandy, M.: The Gesture Pendant: A Selfilluminating, Wearable, Infrared Computer Vision System for Home Automation Control and Medical Monitoring., Proceedings of 4 th International Symposium on Wearable Computers(ISWC'2000), pp. 87-94 (2000).

22. Starner, T., Weaver, J. and Pentland, A.: A Wearable Computer Based American Sign Language Recognizer, Proceedings of First International Symposium on Wearable Computers (ISWC'97), pp. 130-137 (1997).

23. Tan, H. and Pentland, A.: Tactual Displays for Wearable Computing, Personal Technologies, Vol. 1, pp. 225-230 (1997).

24. Tarumi, H., Morishita, K., Nakao, M. and Kambayashi, Y.: SpaceTag: An Overlaid Virtual System and its Application, Proceedings of International Conference on Multimedia Computing and Systems(ICMCS'99), Vol.1, pp. 207-212 (1999).

25. Tsukada, K. and Yasumura, M.: Ubi-Finger: Gesture Input Device for Mobile Use, Proceedings of APCHI 2002, Vol. 1, pp. 388-400 (2002).

26. van Erp, J. B. F.: Tactile Navigation Display, Haptic Human-Computer Interaction, Vol. 2058, Springer Publications, pp. 165-173 (2001).

27. van Veen, H. and van Erp, J. B. F.: Tactile information presentation in the cockpit, Haptic Human-Computer Interaction, Vol. 2058, Springer Publications, pp. 174181 (2001).

28. Waist Measure Belt:

http://www.tepia.or.jp/12th/tenji/virtual/photos/mobile/mobMitu.html (in Japanese).

29. Want, R., Fishkin, K., P., Gujar, A. and Harrison, B., L.: Bridging Physical and Virtual Worlds with Electronic Tags, Proceedings of the ACM Conference on Human Factors in Computing Systems (CHI'99), ACM Press, pp. 370-377 (1999).

30. Yamamoto, T. et. al: Two-dimensional directional perception using vibro-tactile sense, Proceedings of Human Interface Symposium 2002, pp. 21-24 (2002). (in Japanese). 